\title{
Multiple Detector-Row CT in Gastric Cancer Staging: Prospective Study
}

\author{
Lorenzo Bruno ${ }^{*}$, Lucia Barni' ${ }^{1}$, Gaia Masini1 ${ }^{1}$ Sabrina Pacciani ${ }^{1}$, Edvige Lucarelli2 \\ Antonella Masserelli2 , Daniela Tomcykova ${ }^{3}$, Filippo Melli' ${ }^{1}$, Luca Boni ${ }^{3}$, \\ Giancarlo Freschi' ${ }^{1}$ Paolo Bechi ${ }^{1}$ \\ ${ }^{1}$ Surgery and Translational Medicine, University of Florence, Florence, Italy \\ ${ }^{2}$ Diagnostic Imaging 4, Azienda Ospedaliero-Universitaria Careggi, Florence, Italy \\ ${ }^{3}$ Clinical Trials Coordinating Center, Azienda Ospedaliero-Universitaria Careggi, Florence, Italy \\ Email: Ibruno@katamail.com
}

Received 10 October 2014; revised 12 November 2014; accepted 21 November 2014

Academic Editor: Sibu P. Saha, University of Kentucky, USA

Copyright (C) 2014 by authors and Scientific Research Publishing Inc.

This work is licensed under the Creative Commons Attribution International License (CC BY).

http://creativecommons.org/licenses/by/4.0/

(c) (i) Open Access

\begin{abstract}
The aim of this study was to evaluate the accuracy of multiple detector computed tomography (MDCT) in the preoperative staging of gastric cancer, prospectively comparing CT findings with pathological findings at surgery, in a single-center study. A total of 19 consecutive patients with primary cancer recruited between March and July 2014 were submitted to preoperative MDCT staging according to a standard protocol. All diagnostic procedures were performed by dedicated radiologists who were unaware of the final pathological results. Subsequently, 16 patients underwent surgical treatment and 15 were finally included in the study. The primary tumor was detected at CT in all 15 cases. CT results for T staging were in agreement with pathological findings in 12 of 15 cases, with overall accuracy of $80 \%$. Stage-specific sensibility was high for advanced stages (sensibility for $\mathrm{T} 1, \mathrm{~T} 3$, and $\mathrm{T} 4$ resulted $60 \%, 85.7 \%$, and $100 \%$, respectively), while earlier stages showed higher specificity (specificity for T1, T3, and T4 resulted $100 \%, 75 \%$, and $91.7 \%$, respectively). Overall $N$ staging accuracy was $86.7 \%$, with 13 of 15 patients correctly staged. Stagespecific sensibility was $75 \%$ for NO and $100 \%$ for N3, while specificity was $100 \%$ for NO and lower for advanced stages. Accuracy for peritoneal involvement was $\mathbf{1 0 0 \%}$. Our findings show a good performance of the diagnostic protocol performed with MDCT tested in this study.
\end{abstract}

\section{Keywords}

Gastric Cancer, MDCT, Staging, Oncologic Imaging

\footnotetext{
${ }^{*}$ Corresponding author.
}

How to cite this paper: Bruno, L., Barni, L., Masini, G., Pacciani, S., Lucarelli, E., Masserelli, A., Tomcykova, D., Melli, F., Boni, L., Freschi, G. and Bechi, P. (2014) Multiple Detector-Row CT in Gastric Cancer Staging: Prospective Study. Journal of Cancer Therapy, 5, 1438-1449. http://dx.doi.org/10.4236/jct.2014.514145 


\section{Introduction}

Despite a decline in the incidence of gastric cancer (GC), this tumor remains the third cause of cancer-related death in both sexes, the second if we only consider the digestive tract.

In western world, the majority of GC cases are diagnosed in an advanced stage, when clinical evidence of the disease (anemia, weight loss, dysphagia, vomit) is already present [1].

In areas like Italy, where GC's incidence does not justify screening programs, the diagnosis of the tumor is mainly endoscopic: upper endoscopy enables tumor detection in most cases and allows biopsies for histological diagnosis [2].

This diagnostic method is however insufficient when it comes to the evaluation of tumor infiltration, lymph nodes involvement and distant metastases: contrast enhanced CT is still the gold standard for tumor staging, especially after the introduction of spiral technique and multiple detector technique [2].

Other diagnostic methods may have higher sensitivity than CT in T staging: endoscopic ultrasound (EUS) shows high sensitivity for parietal invasion; however it is more invasive and highly dependent from the operator's experience. Moreover, due to the high frequency of the probe (>12 MHz), EUS is unable to evaluate nonperigastric lymph nodes or distant metastases. Thus EUS role is limited to early gastric cancers, evaluated for conservative treatment [3] [4].

The interest in preoperative staging has increased after the introduction of different therapeutical approach to GC based on the stage of the disease: either a more conservative approach or a systemic approach, requires a precise evaluation of disease extent [5].

The purpose of this study is to evaluate the diagnostic performance of contrast enhanced multiple detector CT (MDCT) in the exact TNM tumor staging before surgical treatment, compared with pathological staging after surgery. The study has two main aims: first, the validation of an $\mathrm{N}$-staging method, based on double dimensional cut-off, and secondly the validation of a T-staging method, in agreement with the "New MDCT criteria" suggested by Kim et al. [6]. Secondary aims are the evaluation of the influence of tumor maximal dimension $\left(\mathrm{D}_{\max }\right)$ in the definition of $\mathrm{T}$, the evaluation of differences in $\mathrm{T}$ and $\mathrm{N}$ staging in different Lauren histotypes, and evaluation of MDCT accuracy towards peritoneal involvement.

\section{Materials and Methods}

\subsection{Patients}

Between March and July 2014, 19 consecutive patients (11 males and 8 females) with histologically proven adenocarcinoma of the stomach or the esophagogastric junction, underwent MDCT performed for staging purpose. All patients were diagnosed with primary gastric or esophagogastric junction cancer, confirmed at biopsy during upper endoscopy.

To be included in the study all patients were submitted to surgical procedure within 30 days from the staging CT, in order to obtain a pathological staging comparable to the preoperative evaluation. Surgical procedure, when indicated, was performed on average after 11 days from the staging CT. No patient was submitted to preoperative chemo or radiotherapy.

Of the initial 19 patients, 3 were excluded after CT showed liver metastases. 1 patient presented a tumor of the middle and lower esophagus and was therefore excluded. Finally 15 patients were included in the study.

\subsection{CT: Staging Protocol and Technical Parameters}

CT was performed using 16, 64 or 128 slices machine, with spiral technique. Ten to five minutes before image acquisition, $500 \mathrm{ml}$ of tap water were given orally to the patient fasting for at least five hours, to obtain correct gastric distension: gastric filling was considered adequate when gastric folds were not visible on the scans.

Before starting the exam, $20 \mathrm{mg}$ of scopolamine butylbromide were administered intravenously, to induce hypotonia and minimize peristaltic movements: before administration, contraindications were excluded.

The patient was positioned according to the location of the tumor, supine for neoplasias of the gastric corpus or antrum, prone for neoplasias of the upper third.

After a direct thoraco-abdominal acquisition, non ionic contrast medium (Iopromide $370 \mathrm{mg} / \mathrm{ml}$, Iomeprolo $400 \mathrm{mg} / \mathrm{ml}$ ) was administered proportionally to the patient's weight $(1.5-2 \mathrm{mg} / \mathrm{kg}$ ), followed by administration of $40 \mathrm{ml}$ of saline solution, using a peristaltic semiautomatic pump, at a flow rate of $3-4 \mathrm{ml} / \mathrm{s}$ through an $18 \mathrm{G}$ catheter placed in antecubital vein. 
Image acquisition of upper abdomen was performed during the arterial phase, 35 - 40 seconds after intravenous contrast administration. A second acquisition of entire abdomen, from diaphragmatic dome to pubic symphysis, was performed during portal phase, 70 seconds after contrast injection, and was extended to thorax for complete staging.

To avoid artifacts, patients were taught not to breath during image acquisition.

The following technical parameters were used: beam pitch close to 1 , KvP between 100 and 140, using specific algorithms to lower the dose, and 100 - 500 or 100 - $700 \mathrm{mAs}$, depending on the machine used.

Slice thickness was $2.50-3.00 \mathrm{~mm}$ during acquisition, while reconstruction interval was $1.00-1.25 \mathrm{~mm}$.

\subsection{Image Analysis}

Preoperative images were analyzed prospectively by two dedicated radiologists, who were aware of tumor location as described at endoscopy.

For every patient, tumor location on the short and long axis and macroscopic aspect were described.

Then gastric wall infiltration (T) was evaluated, in agreement with AJCC 7th edition [7] C with reference to the "New MDCT Criteria" by Kim et al. [6] (Table 1), based on the assumption that normal gastric wall has a three-layered aspect at CT images: a thin contrast enhanced mucosal inner layer, a low density middle layer, combination of submucosa and muscolaris propria, and an outer, high attenuation layer, formed by subserosa and serosa.

For every patient, we indicated the presence or absence of the three layered pattern. Maximal dimension of the tumor was measured on the best reconstruction plane.

In the evaluation of nodal involvement, we considered two different cut-offs: depending on tumor location and consequent different lymphatic drainage, we distinguished first (N1) and second level nodes (N2, N3), in agreement with JCGC 2nd English edition [8]. Measuring lymph node's short axis, we considered a cut off value of $\geq 5 \mathrm{~mm}$ for $\mathrm{N} 1$, while for second level nodes the cut-off value was $8 \mathrm{~mm}$. $\mathrm{N}$ staging was then performed in agreement with JCGC 3rd English edition/AJCC 7th edition [7] [9].

CT scans were finally evaluated for distant metastases: particular attention was given to the presence/absence of peritoneal carcinomatosis. If present, the extent of peritoneal involvement was assessed with the Peritoneal Cancer Index (PCI) by Sugarbaker. Also these results were confronted with histopathological evaluation to confirm the presence of peritoneal involvement, while quantitative assessment was only preoperative.

As previously said, CT staging was performed independently by two radiologist: when staging was different between the two observers, images were reevaluated and a consensus staging was reached (Figures 1-3).

\subsection{Pathologic Analysis}

16 out of 19 patients were submitted to surgical procedure; as previously said, one was excluded because the disease was staged as esophageal malignancy.

10 patients underwent total gastrectomy, 2 distal gastric resection and 3 cases received palliative interventions (1 subtotal gastrectomy with transverse colon resection, 1 explorative laparotomy, 1 explorative laparotomy with jejunostomy). A D2 lymphadenectomy was performed in all cases.

Table 1. New MDCT criteria.

\begin{tabular}{cl}
\hline STAGE (depth of invasion) & \multicolumn{1}{c}{ New MDCT criteria } \\
\hline T1a (mucosa) & $\begin{array}{l}\text { Tumor shows enhancement and/or thickening of the inner mucosal layer, as compared to the adjacent } \\
\text { normal mucosal layer, with an intact low-density-stripe layer. }\end{array}$ \\
T1b (submucosa) & $\begin{array}{l}\text { Disruption of the low-density-stripe layer (less than } 50 \% \text { of the thickness) is visualized. } \\
\text { Disruption of the low-density-stripe layer (greater than } 50 \% \text { of the thickness) is visualized without } \\
\text { abutting on the outer, slightly high-attenuating layer. }\end{array}$ \\
T2 (muscolaris propria) & $\begin{array}{l}\text { Discrimination between the enhancing gastric lesion and the outer layer is visually impossible, and a } \\
\text { smooth outer margin of the outer layer or a few small linear strandings in the perigastric fat plane are } \\
\text { visualized. } \\
\text { T3 (subserosa) }\end{array}$ \\
T4a (serosa) & $\begin{array}{l}\text { Anfiltration is visualized. } \\
\text { Obliteration of the fat plane between the gastric lesion and the adjacent organs or direct } \\
\text { T4b (adjacent organs) }\end{array}$
\end{tabular}




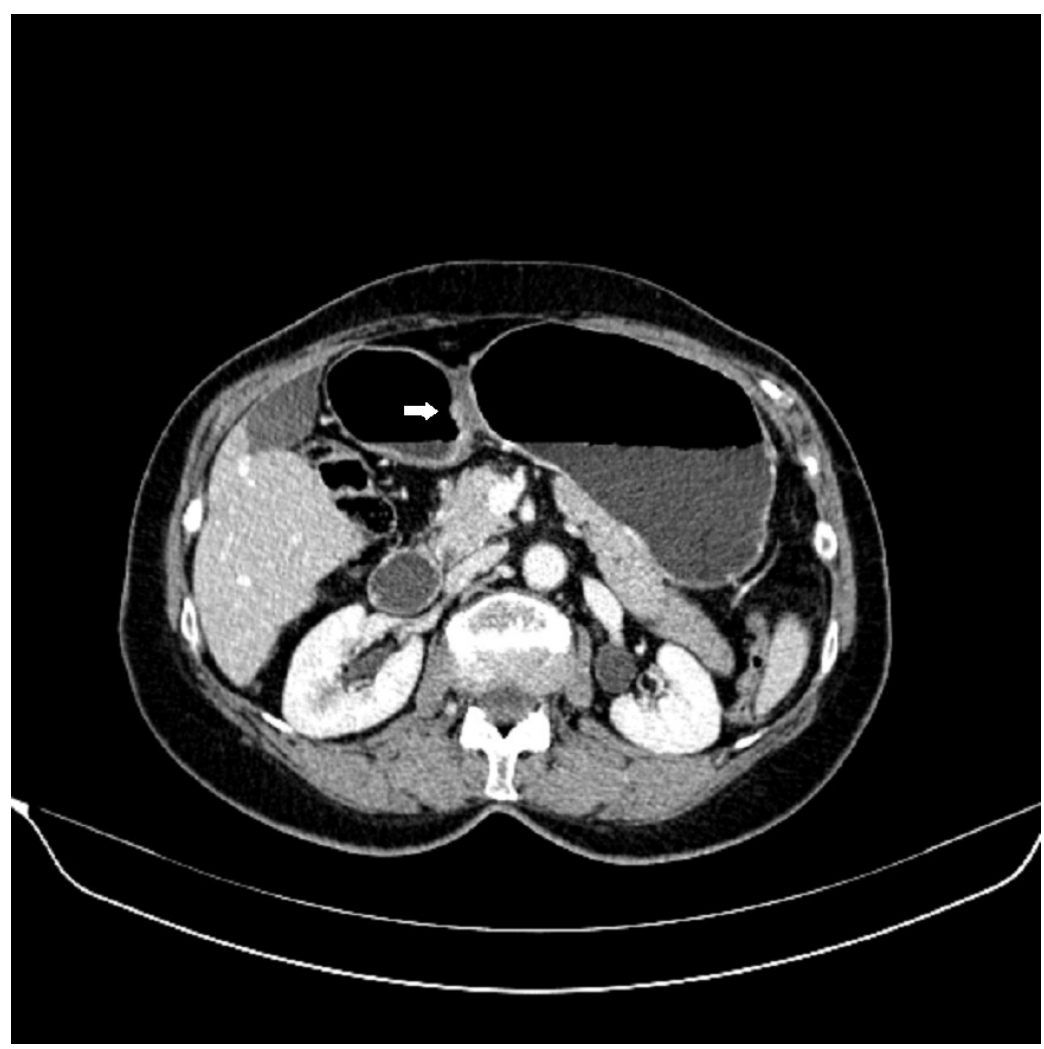

Figure 1. MDCT image in axial plane-T1a tumor.

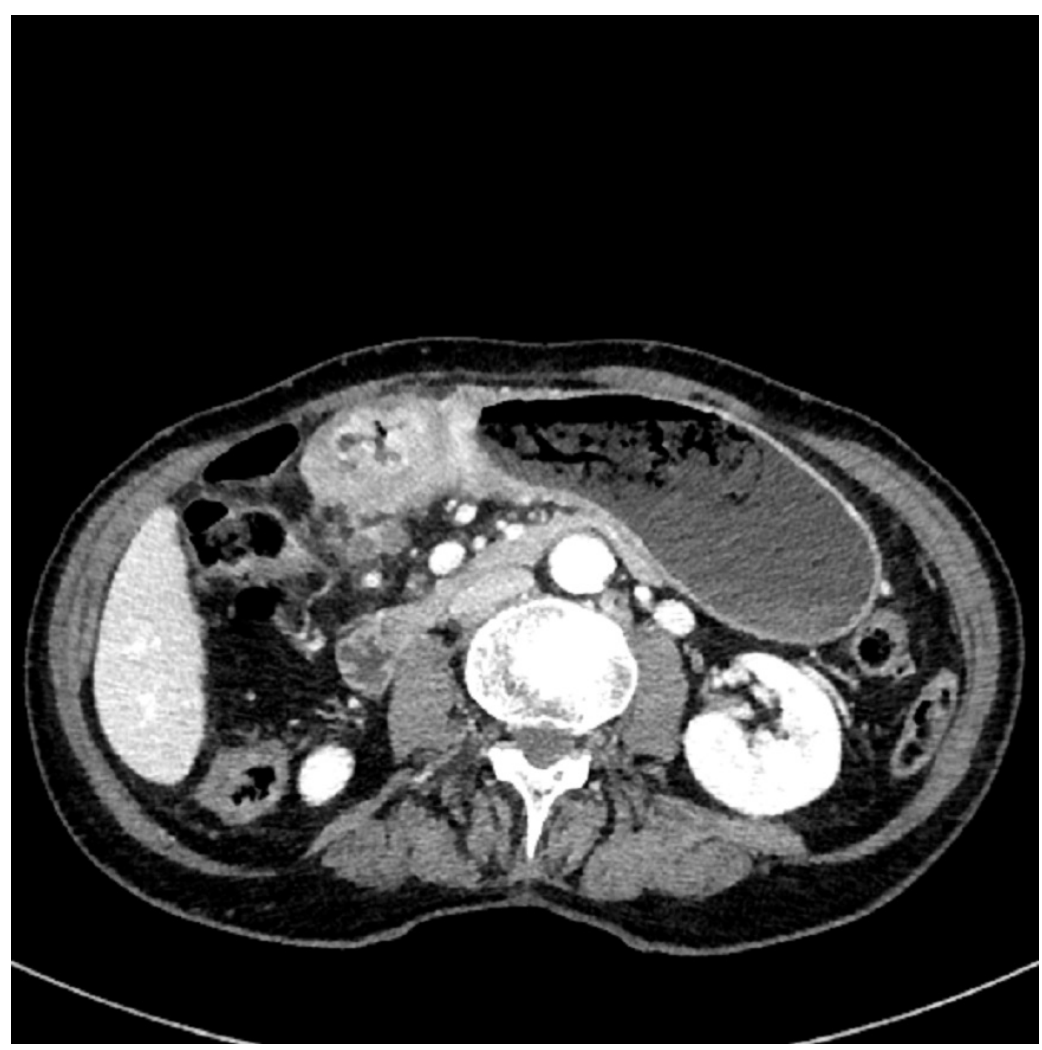

Figure 2. MDCT image in axial plane-T4b tumor with peritoneal involvement. 


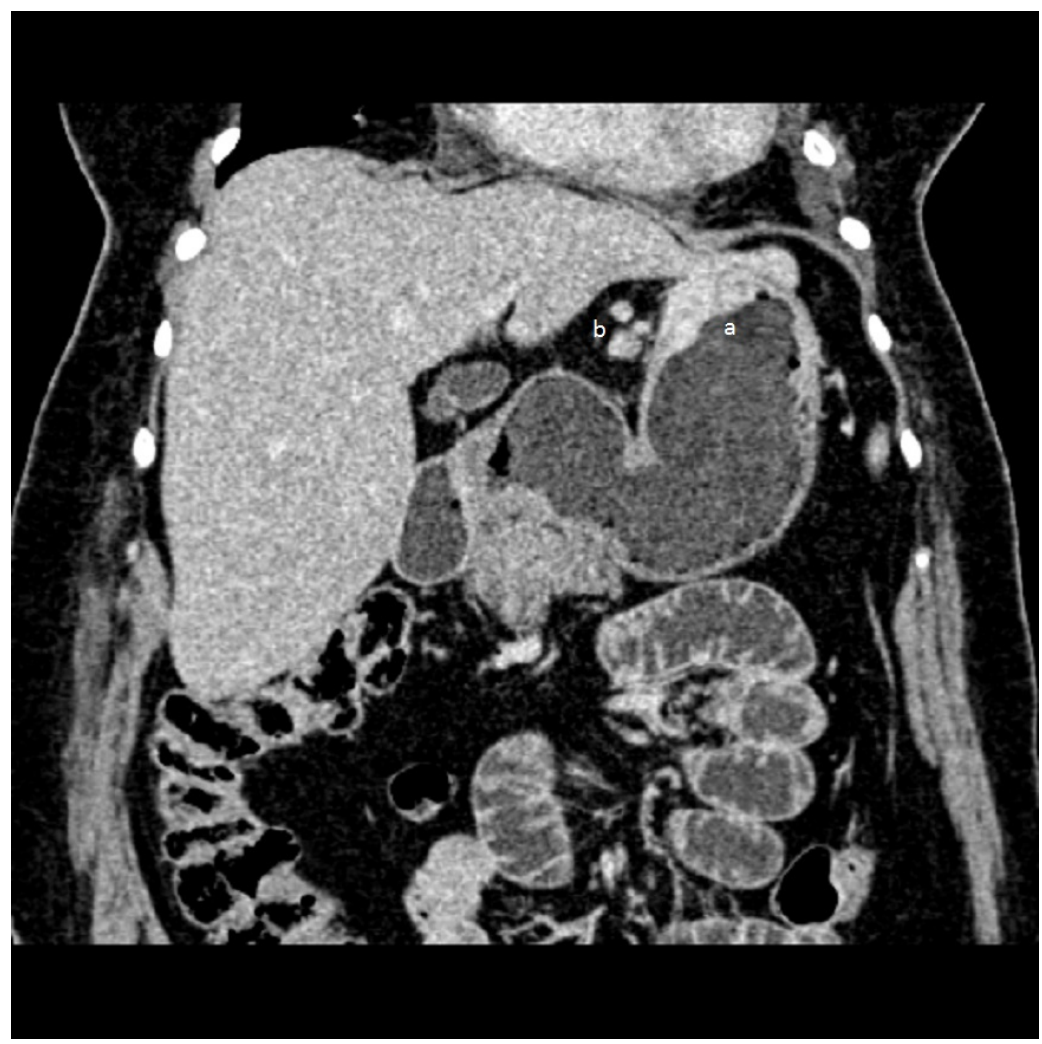

Figure 3. MDCT reconstructed image in coronal-oblique plane-T3 tumor (a) and 3 peritumoral pathological lymph nodes (b).

All the specimens obtained during surgery were analyzed and considered as standard reference for $\mathrm{T}$, $\mathrm{N}$ and peritoneal carcinomatosis staging. In case the tumor was unresectable, intraoperative findings were the reference standard for $\mathrm{T}$, and pathological analysis of lymph nodes, peritoneal nodes and ascitic fluid collected during surgery was used to assess $\mathrm{N}$ and $\mathrm{M}$ parameters.

All specimens were classified following Lauren categories in intestinal type $(n=5)$, diffuse $(n=8)$ and mixed type $(\mathrm{n}=2)$. For statistical analysis, diffuse and mixed types were considered together.

Tumor location, macroscopic aspect and maximal diameter were also evaluated.

\subsection{Statistical Analysis}

Continuous variables were expressed as mean, median, standard deviation.

Statistical analysis was performed on collected data calculating accuracy, sensibility, specificity, positive and negative predictive values and their 95\% confidence interval for each $\mathrm{T}$ and $\mathrm{N}$ stage, for peritoneal involvement and overall staging.

The level of agreement between the two independent radiologists and between CT and pathological staging was measured with weighted Cohen's kappa, for T, N, P and overall staging.

The relationship between certain clinicopathological characteristics (age, gender, tumor location, maximal diameter, histotype, three-layered wall pattern) and CT accuracy for $\mathrm{T}$ and $\mathrm{N}$ was evaluated with chi square test.

A p-value $\leq 0.05$ was considered statistically significant.

\section{Results}

Primary tumor was detected in all 15 cases at CT scan (100\%). Tumor location evaluated on long axis was upper third in 7 patients, middle third in 7 patients, lower third in 1 patient.

The three-layered pattern of the wall was detected in 6 cases: 6 cases had a single-layer aspect and 3 appeared to have 2 layers. 
In all patients, gastric wall thickening (range 7 - 30 mm of thickness) was visible, and in 4 patients there was evidence of lumen obstruction. Wall thickening was more evident in advanced gastric cancers (mean thickness $23.4 \mathrm{~mm}$ ) than in EGC (mean thickness $14.2 \mathrm{~mm}$ ).

Regarding perigastric fat, in 6 cases (5 T1, 1 T3) the outer margin was smooth; in 5 cases (all T3) few small linear strandings were visible, while in the remaining 4 cases $(3 \mathrm{~T} 4,1 \mathrm{~T} 3)$ there was a dense perigastric fat infiltration.

Maximal diameter measured on CT scans was on average $7.34 \pm 3.4 \mathrm{~cm}$, with a mean overestimation of 0.75 $\mathrm{cm}$ in comparison with postoperative measurement.

\subsection{T Staging}

CT staged as follows, 2 T1b and 1 T1a (3 EGC, 20\%); 8 T3, 1 T4a and 3 T4b (12 AGC, 80\%). Weighted Cohen's kappa value showed almost perfect agreement between the two independent observers (weighted kappa $=$ 0.8193) for this parameter. Pathological staging confirmed 12 out of 15 cases, with an overall accuracy of $80 \%$ for T staging. The 3 non congruent cases were all overstaged: 2 T1 overstaged at CT as T3 and 1 T3 overstaged as T4a (Table 2). No patient was understaged. Results of CT diagnostic performance for every stage of $\mathrm{T}$ are shown in Table 3.

Among patients with overstaged EGC, one had Small Mucosal type, according to Kodama's classification, and one was type Pen A. At CT scans, the first showed an irregular thickening (approximately $12 \mathrm{~mm}$ ) of the gastrojejunal anastomosis (stump cancer), extended for about $3 \mathrm{~cm}$, with a single-layer pattern; the patient with Pen A EGC showed a three-layer pattern, in the presence of a polypoid tumor of the gastric antrum measuring $28 \times 22 \mathrm{~mm}$, with patchy contrast enhancement. Both cases showed a clean and smooth outer margin. Correctly staged EGC showed a modest thickening $(7-14 \mathrm{~mm})$, about $1.5-2 \mathrm{~cm}$ in size, with marked enhancement of the inner mucosal layer. In all three correctly staged EGC cases, three-layered pattern was visible.

The case with T3 tumor overstaged T4a appeared at CT images as a stenotic area, extended for $6 \mathrm{~cm}$ in the gastric corpus; three layered pattern was present, but accompanied by a dense band-like aspect of the perigastric fat. The correctly staged T3 cases showed a similar level of wall thickening $(16-30 \mathrm{~mm})$ and tumor size $(5-12$ $\mathrm{cm}$ ), but the outer layer aspect was smooth or showed few small linear strandings.

Three patients were correctly staged as T4b: they showed marked wall thickening (22 - $28 \mathrm{~mm}$ ), with extensive stenosis of the lumen. The gastric wall showed a two-layer aspect, in presence of an irregular appearance of the outer margin and perigastric fat, with infiltration of adjacent organs.

The analysis of the correlation between certain clinicopathological characteristics and CT accuracy in $\mathrm{T}$ staging, showed a significant difference between correctly staged and overstaged tumors in relation to histotype ( $\mathrm{p}=$ 0.0040), while age, gender, tumor location, tumor size and three-layer pattern showed no significant difference (Table 4).

Table 2. Comparison between CT and pathological staging of $\mathrm{T}$.

\begin{tabular}{ccccc}
\hline \multirow{2}{*}{ MDCT staging } & \multicolumn{3}{c}{ Pathological staging } & pT4 \\
\cline { 2 - 5 } T1 & pT1 & pT2 & pT3 & 0 \\
T2 & $3^{\mathrm{a}}$ & 0 & 0 & 0 \\
T3 & 0 & 0 & 0 & 0 \\
T4 & 2 & 0 & $6^{\mathrm{a}}$ & $3^{\mathrm{a}}$ \\
\hline
\end{tabular}

${ }^{\mathrm{a}}$ Cases in which the two staging were congruent.

Table 3. CT diagnostic performance for each T stage.

\begin{tabular}{|c|c|c|c|c|c|c|}
\hline Stage & $\mathbf{n}$ & Accuracy (\%) & Sensibility (\%) & Specificity (\%) & PPV (\%) & NPV (\%) \\
\hline T1 & 5 & 86.7 & 60 & 100 & 100 & 83.S3 \\
\hline $\mathbf{T} 2$ & 0 & 0 & 0 & 0 & 0 & 0 \\
\hline T3 & 7 & 80 & 85.7 & 75 & 75 & 85.7 \\
\hline T4 & 3 & 93.3 & 100 & 91.7 & 75 & 100 \\
\hline
\end{tabular}


Table 4. Relationship between clinicopathological characteristics and CT accuracy in T staging.

\begin{tabular}{|c|c|c|c|}
\hline Clinicopathological characteristics & Group A $(n=12)$ & Group B $(n=3)$ & p-value \\
\hline Gender & & & 0.6013 \\
\hline Male & 6 & 2 & \\
\hline Female & 6 & 1 & \\
\hline Age (years) & $68 \pm 12.16$ & $72.33 \pm 4.6$ & 0.7231 \\
\hline $\mathbf{D}_{\max }$ & & & 0.0701 \\
\hline$>6 \mathrm{~cm}$ & 7 & 0 & \\
\hline$\leq 6 \mathrm{~cm}$ & 5 & 3 & \\
\hline Location & & & $0.6393 \mathrm{~S}$ \\
\hline $\mathrm{U}$ & 6 & 1 & \\
\hline M & 5 & 2 & \\
\hline $\mathrm{L}$ & 1 & 0 & \\
\hline Histotype & & & 0.0040 \\
\hline Intestinal & 2 & 3 & \\
\hline Diffuse/mixed & 10 & 0 & \\
\hline Three-layer pattern & & & 0.2954 \\
\hline Yes & 4 & 2 & \\
\hline No & 8 & 1 & \\
\hline
\end{tabular}

Group A: correctly staged; Group B: overstaged.

\subsection{N Staging}

At CT 6 patients were staged N0, 1 N1, 3 N2 and 5 N3. As for T staging, agreement between the two independent observers was almost perfect (weighted Cohen's kappa $=0.8160$ ).

Pathologic staging performed on lymph nodes obtained during surgery confirmed 13 out of 15 cases (Table $5)$, resulting in an overall accuracy of $86.7 \%$.

Weighted Cohen's kappa measured between CT and pathological staging of N resulted 0.7973, representing a good level of agreement.

Among N0 patients, 6 out of 8 were correctly staged, while 2 were overstaged to N2: accuracy consequently resulted $86.7 \%$, sensibility $75 \%$ and specificity $100 \%$. Because of the two overstaged cases, N2 specificity was 85.7\%. N1 and N3 showed instead high values of sensitivity and specificity (Table 6).

The two overstaged cases had 4 and 6 lymph nodes positive at CT, respectively: the first showed enlarged perigastric nodes, with short axis between 5 and $8 \mathrm{~mm}$ (cut off $5 \mathrm{~mm}$ ). The second patient had 4 perigastric nodes with short axis between 5 and $8 \mathrm{~mm}$ and 2 non perigastric (N2, celiac nodes) with short axis between 8 and 9 $\mathrm{mm}$ (cut off $8 \mathrm{~mm}$ ).

Correlation between clinicopathological characteristics and CT accuracy were evaluated also for N staging: patients' distribution resulted again significantly different for Lauren histotype $(p=0.0246)$, since all overstaged patients had intestinal type tumor (Table 7).

Table 5. Comparison between CT and pathological staging of N.

\begin{tabular}{ccccc}
\hline & & \multicolumn{2}{c}{ Pathological staging } & pN2 \\
MDCT staging & pN0 & pN1 & 0 & 0 \\
\cline { 2 - 5 } N0 & $6^{\mathrm{a}}$ & 0 & 0 & 0 \\
N1 & 0 & $1^{\mathrm{a}}$ & $1^{\mathrm{a}}$ & 0 \\
N2 & 2 & 0 & 0 & $5^{\mathrm{a}}$ \\
N3 & 0 & 0 & 0 \\
\hline
\end{tabular}

${ }^{\mathrm{a} C}$ Cases in which the two staging were congruent. 
Table 6. CT diagnostic performance for each $\mathrm{N}$ stage.

\begin{tabular}{ccccccc}
\hline Stage & $\mathbf{n}$ & Accuracy (\%) & Sensibility (\%) & Specificity (\%) & PPV (\%) & NPV (\%) \\
\hline N0 & 8 & 86.7 & 75 & 100 & 100 & 77.7 \\
N1 & 1 & 100 & 100 & 100 & 100 & 100 \\
N2 & 1 & 86.7 & 100 & 85.7 & 33.3 & 100 \\
N3 & 5 & 100 & 100 & 100 & 100 & 100 \\
\hline
\end{tabular}

Table 7. Relationship between clinicopathological characteristics and CT accuracy in N staging.

\begin{tabular}{|c|c|c|c|}
\hline Clinicopathological characteristics & Group A $(n=13)$ & Group B $(n=2)$ & p-value \\
\hline Gender & & & 0.9192 \\
\hline Male & 7 & 1 & \\
\hline Female & 6 & 1 & \\
\hline Age (years) & $71 \pm 5.66$ & $78.54 \pm 11.80$ & 0.9322 \\
\hline $\mathbf{D}_{\max }$ & & & 0.1553 \\
\hline$>6 \mathrm{~cm}$ & 7 & 0 & \\
\hline$\leq 6 \mathrm{~cm}$ & 6 & 2 & \\
\hline Location & & & 0.1823 \\
\hline $\mathrm{U}$ & 7 & 0 & \\
\hline M & 5 & 2 & \\
\hline $\mathrm{L}$ & 1 & 0 & \\
\hline Histotype & & & 0.0246 \\
\hline Intestinal & 3 & 2 & \\
\hline Diffuse/Mixed & 10 & 0 & \\
\hline
\end{tabular}

Group A: correctly staged; Group B: overstaged.

\subsection{Distant Metastasis and Peritoneal Involvement}

As previously said, 3 of 19 initial patients did not undergo surgical treatment because liver metastases were seen on CT.

Among the 15 patients included in the study, 3 showed ascites and nodular lesions on peritoneal surface: in all three cases, cytological examination of peritoneal fluid and histologic analysis on peritoneal nodules obtained during surgery confirmed the presence of peritoneal carcinomatosis.

The level of agreement between the two radiologist on this parameter was perfect (weighted Cohen's kappa = 1.0000), and so was the agreement between CT and pathological staging (weighted Cohen's kappa $=1.0000$ ) (Table 8).

Quantification of peritoneal involvement evaluated with Sugarbaker's PCI showed scores between 4 and 26. Loculated ascites was present in all three cases.

PCI was not reevaluated intraoperatively, were we only evaluated the area of peritoneal cavity involved, that resulted in agreement with CT evaluation in all three cases.

\subsection{Staging}

Combining $\mathrm{T}, \mathrm{N}$ and $\mathrm{M}$ parameters, we evaluated tumor staging.

Comparing the two radiologists' observations, the level of agreement resulted almost perfect (weighted Cohen's kappa $=0.9412$ ); also agreement between CT and pathological staging showed a good result (weighted Cohen’s kappa $=0.7353$ ).

Overall staging accuracy was $80 \%$. Results were congruent to those obtained for single T, N and M parameters (Table 9). 
Table 8. Comparison between CT and pathological staging.

\begin{tabular}{ccc}
\hline \multirow{2}{*}{ Pathological staging } & PDCT & \\
\cline { 2 - 3 } & $12^{\mathrm{a}}$ & $\mathbf{P}+$ \\
\hline $\mathbf{P}-$ & 0 & 0 \\
$\mathbf{P}+$ & & $3^{\mathrm{a}}$ \\
\hline
\end{tabular}

${ }^{\mathrm{a} C a s e s}$ in which the two staging were congruent.

Table 9. CT staging performance for overall staging.

\begin{tabular}{ccccc}
\hline STAGE & $\mathbf{n}$ & Accuracy (\%) & Sensibility (\%) & Specificity (\%) \\
\hline I & 5 & 86.7 & 60 & 100 \\
II & 4 & 86.7 & 75 & 90.9 \\
III & 3 & 86.7 & 100 & 83.3 \\
IV & 3 & 100 & 100 & 100 \\
\hline
\end{tabular}

\section{Discussion}

An accurate and reliable staging method is an essential requirement in the perspective of a multimodal treatment for GC, mainly based on the stage of the disease.

CT is currently part of the preoperative algorithm for tumor diagnosis and staging, but is considered the gold standard for distant metastases detection rather than a method for $\mathrm{T}$ and $\mathrm{N}$ staging; for these parameters gold standard remains pathological staging after surgery [10].

However, with the growing interest towards a more conservative therapy for EGC and neoadjuvant/perioperative therapy for AGC, preoperative staging becomes an essential feature: to exclude nodal involvement and infiltration beyond the submucosa and to obtain a reference point to evaluate response to preoperative treatment, respectively.

Therefore, the necessity of a staging technique with reasonable diagnostic accuracy, repeatable and non operator-dependent, brought new interest to CT staging, especially after the introduction of multiple detector technique and protocols using stomach distension with neutral (water) or negative (air) distension.

In the present study we used water distension, easier and equally effective than air distension; $500 \mathrm{ml}$ resulted adequate for all patients, even though in case of marked stenosis seen on endoscopy, it can be indicated to give half of the dose to avoid gastric overdistension that can lead to emesis.

Multiple detector CT reduces volume averaging effect and increases spatial resolution.

After contrast medium injection, arterial phase (35 - $40 \mathrm{~s}$ ) is important to detect the primary tumor and evaluate $\mathrm{T}$, while portal phase $(70 \mathrm{~s})$ is useful to assess nodal status and distant metastases. Chen et al. report that a later scan (150 - 180 s) can add information to the assessment of wall infiltration [11].

Standardization is necessary not only in the diagnostic technique, but also in interpreting CT images: to define T staging, we referred to "New MDCT Criteria" by Kim et al. [6], while double dimensional cut off for lymph nodes was established on the basis of a metanalysis on the role of imaging in assessing nodal status in GC [12].

Overall accuracy in T staging was, in our study, 80\%, in agreement with the results obtained by Kim et al. (77\% - 82\%), even though stage-specific accuracy, sensibility and specificity were slightly different, probably because of the small number of the considered sample and also as a result of the different composition of the two populations: in the Korean sample, EGC were almost $60 \%$, while in our study they resulted around $30 \%$.

In our study, sensibility was higher in advanced $\mathrm{T}$ stages, while lower stages showed better specificity: there were no understaged patients. This could result, clinically, in the overtreatment of EGC patients, but given the higher prevalence of a locally advanced disease in western countries compared to eastern population, this can be considered a good diagnostic performance.

Among the two non-correctly staged EGC, one was Small Mucosal according to Kodama and the other was Pen A, type that shows a worse prognosis than other EGCs and has therefore to be correctly diagnosed: however, since both cases were overstaged, the result from a clinical point of view was a radical treatment, as EGC type Pen A requires.

Obtaining a precise distinction between T1a and T1b using CT seems difficult: it can be useful to refer to the 
patient's clinical history (the only T1a patient in our sample presented a tumor diagnosed during a six-month follow up for low-grade dysplasia), but uncertainty could justify the use of EUS in those patients, in addition to MDCT, in the perspective of a conservative treatment with EMR/ESD.

The analysis of patients' distribution regarding different clinicopathological characteristics showed significant difference relatively to histotype, both for $\mathrm{T}$ and $\mathrm{N}$ staging: this outcome agrees with the results obtained by Marrelli et al. regarding paraortic nodes (PAN) [13], reporting that all patients with false positive lymph nodes resulted intestinal type.

Also in our study the three overstaged tumors were all intestinal type. The hypothesis is that intestinal type is associated with a higher grade of inflammation and fibrosis compared to diffuse/mixed type; also the expansive growth pattern that characterizes this histotype could influence the correct distinction of T stages.

Regarding nodal involvement, we noted that the two pN0 cases overstaged to N2 had a short axis of false positive nodes between 5 and $8 \mathrm{~mm}$, while in the only correctly staged intestinal type patient, short axis of true positive nodes was 14 and $11 \mathrm{~mm}$. Similar results are reported by Marrelli et al. about PAN.

According to these findings, we could consider using different dimensional cut offs depending on tumor histotype: to this end, defining histotype before pathological examination is necessary. The possibility to obtain this information from the analysis of endoscopical biopsies is limited by tumor heterogeneity and endoscopy inaccuracy in collecting only neoplastic tissue: as for the analysis of HER2 expression, 5 to 6 bioptical samples of neoplastic tissue are necessary to define histotype [14] [15].

Regarding maximal diameter of the tumor, it did not result significantly different between correctly staged and overstaged patients; moreover, maximal diameter measurement had about $1 \mathrm{~cm}$ overestimation at CT compared to pathological measurement: this could either be the consequence of measurement at CT of certain areas that are not macroscopically evident on surgical specimen, or the effect of formalin fixation, that is reported to cause up to $20 \%$ - $30 \%$ specimen shrinkage [16].

The presence of the three-layer pattern was not significantly different in the two groups, but 4 out of $5 \mathrm{~T} 1$ cases showed this aspect while only 2 out of 8 T3/T4 patients had a three-layered gastric wall at CT: three-layer pattern is significantly $(\mathrm{p}=0.0253)$ more frequent among EGC cases, feature that can help in image interpretation.

Also for $\mathrm{N}$ parameters the protocol showed a good diagnostic performance: receiver operating characteristics (ROC) analysis showed that with the considered cut-off values (5 mm for N1, $8 \mathrm{~mm}$ for N2-N3), accuracy in nodal status evaluation was high, with an area under curve between 0.87 and 1.

Particularly relevant are the high sensibility and negative predictive value (NPV) in the presence of nodal involvement (N1, N2, N3): this can result clinically in an overtreatment of overstaged patients, but for T1N0 patients, candidates for conservative treatment, high positive predictive value (PPV) of N0 and high NPV of stages higher than N1, allow to exclude nodal involvement.

These results suggest that these cut off values could be reasonably used also in the diagnosis of PAN metastases, even though the limited number of patients in our study does not permit definite conclusions. Similar results were reported by Marrelli et al. particularly for PAN, with 97\% NPV, using the same $8 \mathrm{~mm}$ cut off that was considered in the present study [13].

CT accuracy in detecting PAN involvement is mainly important for neoadjuvant therapy: it could allow patient selection for this treatment and could be useful in evaluating response to chemotherapy [13].

For peritoneal involvement, CT accuracy was $100 \%$ : this result agrees with the outcome of various studies, were CT showed $93 \%$ accuracy [17]. We also quantified peritoneal involvement with PCI, score mainly used to measure carcinomatosis in the perspective of a treatment with cytoreductive surgery and HIPEC (Hyperthermic Intraperitoneal Chemotherapy), especially in ovarian cancer.

The reference standard to compare CT staging of PCI is exploratory laparoscopy. However, for GC treatment, where the use of cytoreductive surgery and HIPEC is still controversial, we consider CT accuracy in peritoneal involvement detection more relevant, in order to select patients that are not candidates neither for surgery, excepting palliative surgery, nor neoadjuvant therapy.

\section{Conclusions}

In order to perform specific therapy for carcinomatosis, staging gold standard remains laparoscopy. In conclusion, the results of the present study are indicative of a good diagnostic performance of the staging protocol for GC with MDCT tested in the present study. 
The analysis of the level of agreement between the two independent observers, almost perfect, shows that the diagnostic examination and image analysis are highly repeatable.

High sensibility and NPV for advanced T and N stages avoid understaging, while high specificity and high PPV of T1 and N0 reduce false positives for lower stages.

High accuracy was shown also in peritoneal involvement detection. The study also shows that intestinal histotype is significantly more associated with $\mathrm{T}$ and $\mathrm{N}$ overstaging: definition of different cut off values on the basis of tumor histotype could be considered. Limits to the study are mainly represented by the small sample, although recruited in a short period of time and managed homogeneously.

Further studies are necessary to confirm the results on a higher scale; however, our results appear to validate the use of MDCT as a staging method in neoadjuvant therapy protocols.

\section{References}

[1] Ferlay, J., Soerjomataram, I., Ervik, M., Dikshit, R., Eser, S., Mathers, C., Rebelo, M., Parkin, D.M., Forman, D. and Bray, F. (2012) GLOBOCAN 2012 v1.0, Cancer Incidence and Mortality Worldwide: IARC Cancer Base No. 11. GLOBOCAN. http://globocan.iarc.fr

[2] Decourcy Hallinan, J.T.P. and Venkatesh, S.K. (2013) Gastric Carcinoma: Imaging, Diagnosis, Staging and Assessment of Treatment Response. Cancer Imaging, 13, 212-227. http://dx.doi.org/10.1102/1470-7330.2013.0023

[3] Kwee, R.M. and Kwee, T.C. (2007) Imaging in Local Staging of Gastric Cancer: A Systematic Review. Journal of Clinical Oncology, 25, 2107-2116. http://dx.doi.org/10.1200/JCO.2006.09.5224

[4] Asano, M. (2012) Endoscopic Submucosal Dissection and Surgical Treatment for Gastrointestinal Cancer. World Journal of Gastrointestinal Endoscopy, 4, 438-447. http://dx.doi.org/10.4253/wjge.v4.i10.438

[5] Xu, A.M., Huang, L., Liu, W., Gao, S., Han, W.X. and Wei, Z.J. (2014) Neoadjuvant Chemotherapy Followed by Surgery versus Surgery Alone for Gastric Carcinoma: Systematic Review and Meta-Analysis of Randomized Controlled Trials. PLoS ONE, 9, e86941. http://dx.doi.org/10.1371/journal.pone.0086941

[6] Kim, J.W., Shin, S.S., Heo, S.H., Choi, Y.D., Lim, H.S., Park, Y.K., Park, C.H., Jeong, Y.Y. and Kang, H.K. (2012) Diagnostic Performance of 64-Secion CT Using CT Gastrography in Preoperative T Staging of Gastric Cancer According to 7th Edition of AJCC Cancer Staging Manual. European Radiology, 22, 654-662. http://dx.doi.org/10.1007/s00330-011-2283-3

[7] Washington, K. (2010) 7th Edition of the AJCC Cancer Staging Manual: Stomach. Annals of Surgical Oncology, 17, 3077-3079. http://dx.doi.org/10.1245/s10434-010-1362-z

[8] Japanese Gastric Cancer Association (1998) Japanese Classification of Gastric Carcinoma. 2nd English Edition. Gastric Cancer, 1, 10-24. http://dx.doi.org/10.1007/PL00011681

[9] Japanese Gastric Cancer Association (2011) Japanese Classification of Gastric Carcinoma. 3rd English Edition. Gastric Cancer, 14, 101-112. http://dx.doi.org/10.1007/s10120-011-0041-5

[10] Chua, Y.L. and Cunningham, D. (2007) The UK NCRI MAGIC Trial of Preoperative Chemotherapy in Resectable Gastric Cancer: Implications for Clinical Practice. Annals of Surgical Oncology, 14, 2687-2690. http://dx.doi.org/10.1245/s10434-007-9423-7

[11] Chen, C.Y., Hsu, J.S., Wu, D.C., Kang, W.Y., Hsieh, J.S., Jaw, T.S., Wu, M.T. and Liu, G.C. (2007) Gastric Cancer: Preoperative Local Staging with 3D Multi-Detector Row CT-Correlation with Surgical and Histopatologic Results. Gastrointestinal Imaging, 242, 472-482.

[12] Kwee, R.M. and Kwee, T.C. (2009) Imaging in Assessing Lymph Node Status in Gastric Cancer. Gastric Cancer, 12, 6-22. http://dx.doi.org/10.1007/s10120-008-0492-5

[13] Marrelli, D., Mazzei, M.A., Pedrazzani, C., Di Martino, M., Vindigni, C., Corso, G., Morelli, E., Volterrani, L. and Roviello, F. (2011) High Accuracy of Multislices Computed Tomography (MSCT) for Para-Aortic Lymph-Node Metastases from Gastric Cancer: A Prospective Single-Center Study. Annals of Surgical Oncology, 18, 2265-2272. http://dx.doi.org/10.1245/s10434-010-1541-y

[14] Ruschoff, J., Hanna, W., Bilous, M., Hofmann, M., Osamura, R.Y., Penault-Llorca, F., van de Vijver, M. and Viale, G. (2012) HER2 Testing in Gastric Cancer: A Practical Approach. Modern Pathology, 25, 637-650. http://dx.doi.org/10.1038/modpathol.2011.198

[15] Michel, P., Carrere, N., Lefort, C., Pezet, D., Rebischung, C., Vendrely, V. and Ychou, M. (2014) Cancer de l'estomac. Thésaurus National de Cancérologie Digestive. http://www.tncd.org/

[16] Goldtein, N.S., Soman, A. and Sacksner, J. (1999) Disparate Surgical Margin Lengths of Colorectal Resection Specimens between in Vivo and in Vitro Measurements. The Effects of Surgical Resection and Formalin Fixation on Organ Shrinkage. American Journal of Clinical Pathology, 111, 349-351. 
[17] Mazzei, M.A., Khader, L., Cirigliano, A., Cioffi Squitieri, N., Guerrini, S., Forzoni, B., Marrelli, D., Roviello, F., Mazzei, F.G. and Volterrani, L. (2013) Accuracy of MDCT in the Preoperative Definition of Peritoneal Cancer Index (PCI) Patients with Advanced Ovarian Cancer Who Underwent Peritonectomy and Hypertermic Intraperitoneal Chemotherapy (HIPEC). Abdomi Imaging, 38, 1422-1430. http://dx.doi.org/10.1007/s00261-013-0013-9 
Scientific Research Publishing (SCIRP) is one of the largest Open Access journal publishers. It is currently publishing more than 200 open access, online, peer-reviewed journals covering a wide range of academic disciplines. SCIRP serves the worldwide academic communities and contributes to the progress and application of science with its publication.

Other selected journals from SCIRP are listed as below. Submit your manuscript to us via either submit@scirp.org or Online Submission Portal.
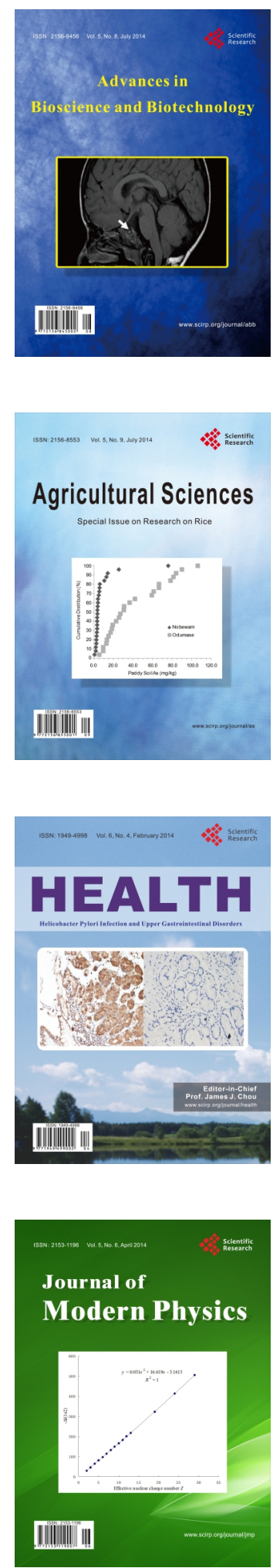
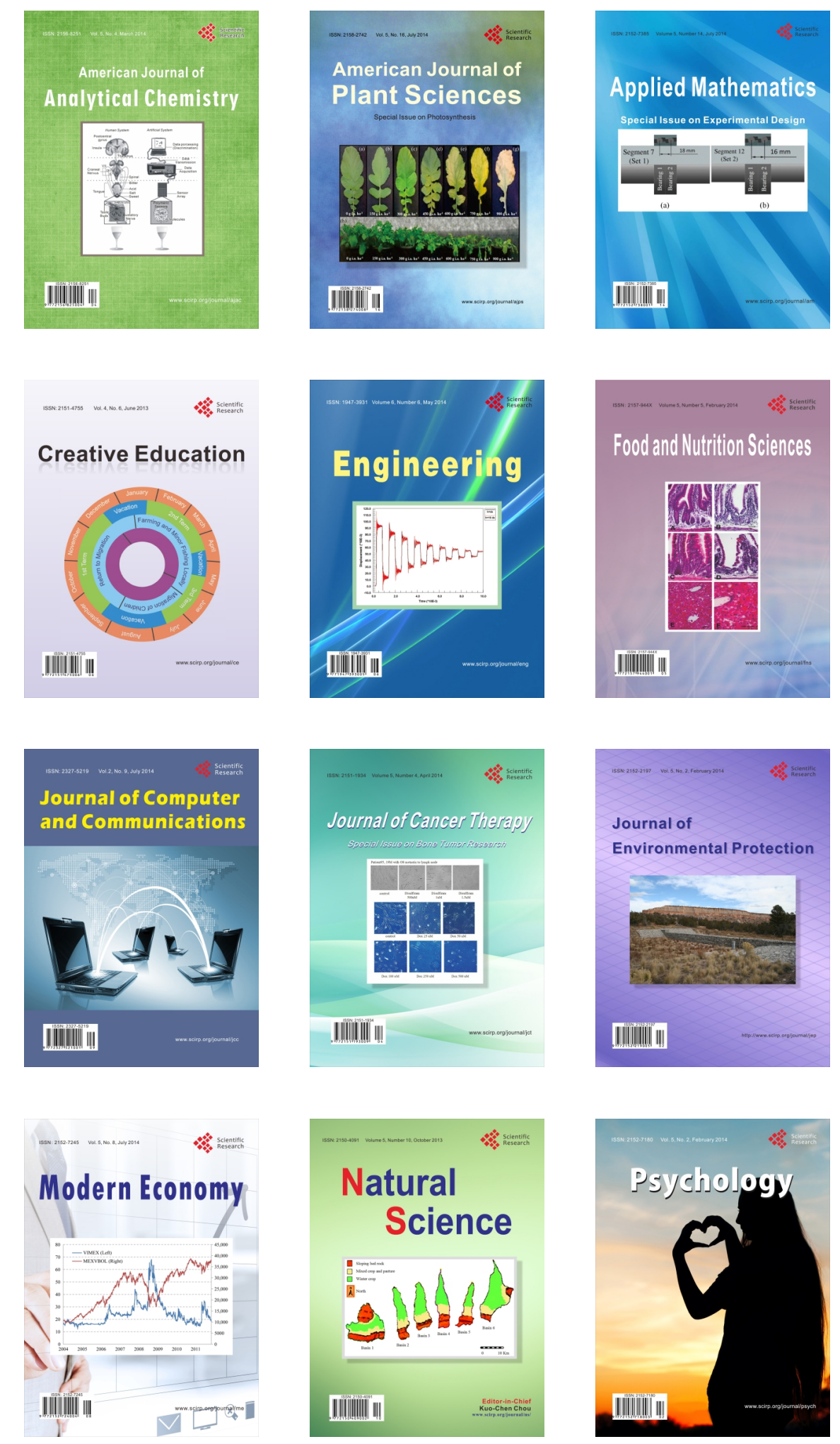